\title{
A Tree Distance Function Based on Multi-sets
}

\author{
Arnoldo José Müller-Molina, Kouichi Hirata, and Takeshi Shinohara \\ Department of Artificial Intelligence, Kyushu Institute of Technology \\ Kawazu 680-4 Iizuka 820-8502, Japan \\ arnoldo@daisy.ai.kyutech.ac.jp \\ \{hirata, shino\}@ai.kyutech.ac.jp
}

\begin{abstract}
We introduce a tree distance function based on multi-sets. We show that this function is a metric on tree spaces, and we design an algorithm to compute the distance between trees of size at most $n$ in $O\left(n^{2}\right)$ time and $O(n)$ space. Contrary to other tree distance functions that require expensive memory allocations to maintain dynamic programming tables of forests, our function can be implemented over simple and static structures. Additionally, we present a case study in which we compare our function with other two distance functions.
\end{abstract}

Key words: Tree edit distance, Program matching, Triangle inequality, Metric

\section{Introduction}

Analysis of tree structured data is required in many fields $[6,9,18]$. Our research group is particularly interested in the field of approximate binary program matching for the detection of Open Source/Libre software license violations $[11,12]$. A recent technique in this field works by generating fragments, that are regarded as trees, from control flow graphs. By means of a tree distance function, the similarity of two programs can be computed. We are interested in distance functions that satisfy the triangle inequality. If this property is satisfied, then we can employ dimension reduction techniques like SMAP [14], and therefore matching speed on massive databases improves considerably. Additionally, it is desirable that the distance function is a metric. Specifically, it is necessary for the distance function to satisfy the property: $d(x, y)=0$ if and only if $x=y$. If this property is satisfied, the matching of programs is "safer" and false positives are reduced.

An overview of current literature [2] presents a tree edit distance function and several variants. To compute every distance function that is metric in [2], it is necessary at least $O\left(n^{2}\right)$ time where $n$ is the maximum number of nodes in two given trees. In this paper, we propose a new tree distance function based on multi-sets that is simple to compute, and that does not require dynamic programming or intensive memory allocations. The main contribution of this paper is to introduce a metric for rooted ordered labeled trees that can be computed in $O\left(n^{2}\right)$ time and $O(n)$ space. We denote this metric as $m t d$. The 
rest of the paper is organized as follows. Section 2 introduces related works. In Section 3, the proposed distance $m t d$ is described and in Section 4, a naive algorithm to compute $m t d$ is presented. Finally in Section 5, we present a case study.

\section{Related Works}

The generally accepted similarity measure for trees is the tree-edit distance metric (ted) introduced by Tai[15] in 1979. Klein[10] proposed an $O\left(n^{3} \log (n)\right)$ algorithm to compute ted. This problem is known to be NP-hard for unordered trees[19]. Currently, the fastest algorithm for ordered trees is $O\left(n^{3}\right)$ [5].

Chawathe et al.[3] introduced an approach in which the set of edit operations is extended. This distance is NP-complete, and the fastest heuristic runs in $O\left(n^{3}\right)$ time. A different variation [4] is linear, however it is not clear if these similarity functions are metrics nor if they satisfy the triangle inequality.

The tree alignment distance introduced by Jiang et al.[7] is not a metric. Finally, the fastest metric that we are aware of is the constrained edit distance[17], with time $O\left(n^{2}\right)$ and space $O(n \log (n))$. The distance functions described above can be seen as an edit script minimization problem. On the other hand, functions based on $q$-grams $[1,13,16]$ are a vector feature distance minimization. These functions have linear complexity, but they are not metrics.

\subsection{Approximate Program Matching}

The function presented in this paper, is intended to be used in the context of approximate binary program matching field. Müller-Molina and Shinohara introduced a technique based on extracting program fragments [11] from binary programs. A program fragment is a tree whose nodes are machine-level instruction codes. They are extracted from program control flow graphs. By analyzing the frequency distribution of the fragments, it is possible to employ "standard" information retrieval techniques to rank programs by similarity. The technique also uses tree distance functions to find similar pairs of program fragments. In this way, even if fragment normalization rules fail, the distance function can compensate for differences introduced by a program transformation. This process is analogous to the stemming process web information retrieval engines apply to each word of a natural language query. The distance introduced in this paper has

been employed successfully in this context, but its properties were not studied formally.

\section{Tree Similarity Distance}

A tree is a connected graph without cycles. For a tree $T=(V, E)$, we sometimes denote $v \in T$ instead of $v \in V$, and $|T|$ instead of $|V|$. A rooted tree is a tree with one node $r$ chosen as its root. 
Let $r$ be a root of $T$ and $v$ a node in $T$. Then, the parent of $v(\neq r)$ is its adjacent node in a path from $v$ to $r$ in $T$, and the ancestors of $v(\neq r)$ are the nodes contained in a path from the parent of $v$ to $r$ in $T$. The parent and the ancestors of the root $r$ are undefined. We say that $u$ is a child of $v$ if $v$ is the parent of $u$, and $u$ is a descendant of $v$ if $v$ is an ancestor of $u$. A leaf is a node having no children. Furthermore, a complete subtree of $T$ at $v$ is a subtree of $T$ of which its root is $v$ and that contains all descendants of $v$.

A rooted tree $T=(V, E)$ is labeled (by an alphabet $\Sigma$ of labels) if there exists an onto function $l: V \rightarrow \Sigma$ such that $l(v)=a(v \in V, a \in \Sigma)$. A tree is ordered if a left-to-right order for the children of each node is given; unordered otherwise. In this paper, we call a rooted labeled ordered tree simply by a tree.

Next, we introduce the notions of multi-sets. Intuitively, a multi-set is a set that allows an element to occur more than once. The multiplicity $m_{A}(x)$ of an element $x$ in a multi-set $A$ is the number of the occurrences of $x$ in $A$. For a (standard) set $A$, it holds that $m_{A}(x)=1$ for every $x \in A$. It is clear that $x \notin A$ if $m_{A}(x)=0$. The set view $v(A)$ of a multi-set $A$ is a set $\left\{x \in A \mid m_{A}(x) \geq 1\right\}$. For example, for a multi-set $A=\{a, a, a, b, c, c\}$, it holds that $v(A)=\{a, b, c\}$. Additionally, the cardinality $|A|$ of a multi-set $A$ is defined as $\sum_{x \in v(A)} m_{A}(x)$.

We now introduce the multi-set operations. Let $A$ and $B$ be multi-sets. Then, the intersection $A \sqcap B$, the union $A \sqcup B$ and the additive union $A \uplus B$ of $A$ and $B$ are defined as follows.

$$
\begin{aligned}
& A \sqcap B=\left\{x \in v(A) \cap v(B) \mid m_{A \sqcap B}(x)=\min \left\{m_{A}(x), m_{B}(x)\right\}\right\}, \\
& A \sqcup B=\left\{x \in v(A) \cup v(B) \mid m_{A \sqcap B}(x)=\max \left\{m_{A}(x), m_{B}(x)\right\}\right\}, \\
& A \uplus B=\left\{x \in v(A) \cup v(B) \mid m_{A \uplus B}(x)=m_{A}(x)+m_{B}(x)\right\} .
\end{aligned}
$$

For example, let $A=\{a, a, b, c, c\}$ and $B=\{a, b, b, c\}$. Then, it holds that $A \sqcap B=\{a, b, c\}, A \sqcup B=\{a, a, b, b, c, c\}$ and $A \uplus B=\{a, a, a, b, b, b, c, c, c\}$.

Based on the previous operations, we define a similarity measure for multisets:

$$
\delta(A, B)=|A \sqcup B|-|A \sqcap B| .
$$

Finally, we introduce the function $s(T)$ which is the multi-set of all complete subtrees of $T$. For example, in Figure 1 the tree $C(B)$ is not a complete subtree of $T_{1}$, but $C(B, E)$ is a complete subtree of $T_{1}$. The function $n(T)$, is the multi-set of all the nodes of $T$.

\subsection{Distance definition}

The first step to calculate our similarity function is to convert a tree into two multi-sets, one multi-set of complete subtrees and another multi-set that contains all the nodes (without children) of the tree. This can be achieved by invoking functions $s$ and $n$. Once the multi-sets of the trees have been generated, the function $m t d$ can be computed. The function $m t d$ is defined as:

$$
d_{s}\left(T_{1}, T_{2}\right)=\delta\left(s\left(T_{1}\right), s\left(T_{2}\right)\right),
$$




$$
\begin{gathered}
d_{n}\left(T_{1}, T_{2}\right)=\delta\left(n\left(T_{1}\right), n\left(T_{2}\right)\right), \\
\operatorname{mtd}\left(T_{1}, T_{2}\right)=\frac{d_{s}\left(T_{1}, T_{2}\right)+d_{n}\left(T_{1}, T_{2}\right)}{2} .
\end{gathered}
$$

The $m t d$ function is composed of two different distance operations: $d_{s}$ and $d_{n}$. The first function $d_{s}$ is a measure based on the number of equal complete subtrees between $T_{1}$ and $T_{2}$. Note that a change of only one leaf node can have multiple repercussions in all its parents. In general, this measure is very sensitive to changes and quickly the intersection $s\left(T_{1}\right) \sqcap s\left(T_{2}\right)$ becomes void. Nevertheless, an important reason to have this intersection is that it makes sure that $\operatorname{mtd}\left(T_{1}, T_{2}\right)=0$ iff $T_{1}=T_{2}$. Additionally, trees that share many common complete subtrees will receive a high score.

The second function $d_{n}$ is a measure that is likely to find matches since only individual nodes are considered. Note that $d_{n}$ is Kailing's distance based on the label histogram [8]. Using only this measure would make trees very similar to each other, however it is necessary to balance the strictness of $d_{s}$.

To summarize, the intuitive idea of our matching procedure is that a very strict matching $\left(d_{s}\right)$ combined with a permissive matching $\left(d_{n}\right)$ brings a balance to the scoring technique. In the context of binary program matching, we have found that both $m t d$ and $d_{s}$ return acceptable results. Function $m t d$ produces slightly better results than $d_{s}$.

Note that $m t d$ always returns a natural number. Precisely, we have the following proposition:

Proposition 1. $d_{s}\left(T_{1}, T_{2}\right)+d_{n}\left(T_{1}, T_{2}\right)$ is even for any trees $T_{1}$ and $T_{2}$.

Proof. For multi-sets $A$ and $B$, since $|A \sqcup B|+|A \sqcap B|=|A \uplus B|$, it holds that $|A \sqcup B|-|A \sqcap B|=|A \uplus B|-2|A \sqcap B|$. Then, for multi-sets $s\left(T_{i}\right)$, and $n\left(T_{i}\right)$ $(i=1,2)$, the following equation holds:

$$
\begin{aligned}
& \left|s\left(T_{1}\right) \sqcup s\left(T_{2}\right)\right|-\left|s\left(T_{1}\right) \sqcap s\left(T_{2}\right)\right|+\left|n\left(T_{1}\right) \sqcup n\left(T_{2}\right)\right|-\left|n\left(T_{1}\right) \sqcap n\left(T_{2}\right)\right| \\
= & \left|s\left(T_{1}\right) \uplus s\left(T_{2}\right)\right|-2\left|s\left(T_{1}\right) \sqcap s\left(T_{2}\right)\right|+\left|n\left(T_{1}\right) \uplus n\left(T_{2}\right)\right|-2\left|n\left(T_{1}\right) \sqcap n\left(T_{2}\right)\right|
\end{aligned}
$$

Since $\left|s\left(T_{i}\right)\right|=\left|n\left(T_{i}\right)\right|$, let $\left|n\left(T_{i}\right)\right|=\left|s\left(T_{i}\right)\right|=k_{i}$. Hence, we can obtain the following even expression:

$$
2 k_{1}+2 k_{2}-2\left|s\left(T_{1}\right) \sqcap s\left(T_{2}\right)\right|-2\left|n\left(T_{1}\right) \sqcap n\left(T_{2}\right)\right| .
$$

In what follows, we give some examples of $m t d$.

In Figure 1 three examples are displayed. In the first example, $m t d\left(T_{1}, T_{2}\right)$, returns 2 because it does not consider in which place a complete subtree is found (multi-sets do not record the position of the subtrees). The function $\operatorname{ted}\left(T_{1}, T_{3}\right)$ returned 2 because " $\mathrm{C}$ " is deleted, and a new "C" node is inserted over the two "B" nodes.

In the second example, $\operatorname{mtd}\left(T_{1}, T_{3}\right)$ is calculating the distance of two node renaming operations. The result is again the same for $\operatorname{ted}\left(T_{1}, T_{3}\right)$. In the third 
example, $\operatorname{mtd}\left(T_{4}, T_{5}\right)$ returns 5 because all of the complete subtrees of $T_{4}$ except "E" and "F" are not in $T_{5}$. The deeper the modification is, the greater the distance will be. In the context of binary program matching[12] this behavior is desirable because changes in the deepest part of an expression are likely to change more drastically the "meaning" of the expression than changes in upper layers of the expression.

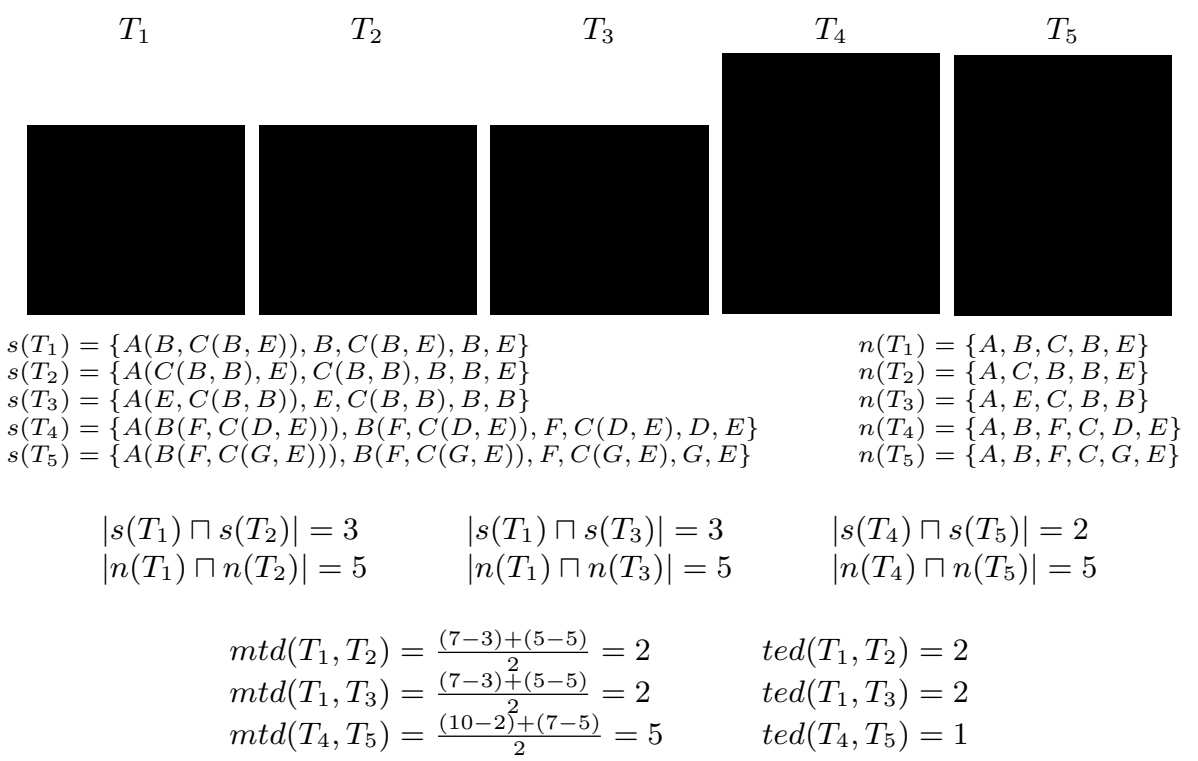

Fig. 1. This example calculates $m t d\left(T_{1}, T_{2}\right), m t d\left(T_{1}, T_{3}\right)$, and $m t d\left(T_{4}, T_{5}\right) . T_{1}$ and $T_{2}$ illustrate the cost of complete subtree movement. $T_{1}$ and $T_{3}$, illustrate two label modification operations. $T_{3}$ and $T_{4}$ depict the cost of one node modified at deep levels of the tree.

In Figure 2, an example in which mtd returns a smaller value than ted is shown. In this case, $\operatorname{mtd}\left(T_{1}, T_{2}\right)$ returns a smaller distance than $\operatorname{ted}\left(T_{1}, T_{2}\right)$ because swapped nodes (rooted in "C" and "D") were children of a node whose label was modified. Because the algorithm does not differentiate when the root node and the children are modified, the score is lower. The function $\operatorname{ted}\left(T_{1}, T_{2}\right)$ returns 3 because "A" is renamed by " $\mathrm{H}$ ", " $\mathrm{D}$ " is deleted, and finally, " $\mathrm{D}$ " is inserted to the left of "C".

Proposition 2. For any trees $T_{1}, T_{2}$ and $T_{3}$, the following statements hold:
1. $\operatorname{mtd}\left(T_{1}, T_{2}\right) \geq 0$
2. $\operatorname{mtd}\left(T_{1}, T_{2}\right)=\operatorname{mtd}\left(T_{1}, T_{2}\right)$
non-negativity
3. $\operatorname{mtd}\left(T_{1}, T_{2}\right)=0 \Longleftrightarrow T_{1}=T_{2}$
symmetry
4. $\operatorname{mtd}\left(T_{1}, T_{3}\right) \leq \operatorname{mtd}\left(T_{1}, T_{2}\right)+\operatorname{mtd}\left(T_{2}, T_{3}\right)$ triangle inequality 


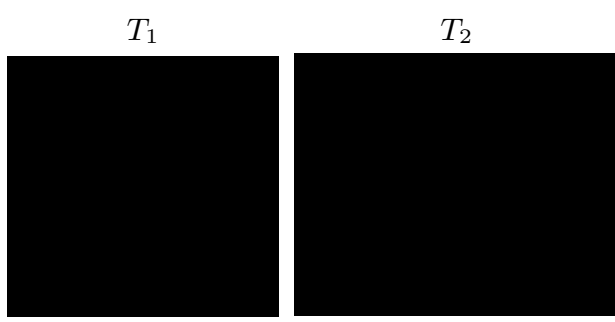

$s\left(T_{1}\right)=\{A(B, C(E, F(G)), D), B, C(E, F(G)), E, F(G), G, D\} \quad n\left(T_{1}\right)=\{A, B, C, E, F, G, D\}$ $s\left(T_{2}\right)=\{H(B, D, C(E, F(G))), B, D, C(E, F(G)), E, F(G), G\} \quad n\left(T_{2}\right)=\{H, B, D, C, E, F, G\}$

$$
\begin{array}{ll}
\left|s\left(T_{1}\right) \sqcap s\left(T_{2}\right)\right|=6 & \left|n\left(T_{1}\right) \sqcap n\left(T_{2}\right)\right|=6 \\
m t d\left(T_{1}, T_{2}\right)=\frac{(8-6)+(8-6)}{2}=2 & \operatorname{ted}\left(T_{1}, T_{2}\right)=3
\end{array}
$$

Fig. 2. In this example, $m t d$ returns a smaller distance than ted.

Proof. Properties 1 and 2 are obvious. Property 3 can be deduced from the fact that $T_{1}$ and $T_{2}$ are returned by the $d_{s}$ function. If there is any change between the trees, the original tree will not be matched in the intersection operation and a distance greater than zero will be computed.

We now prove property 4 . It is sufficient to prove that $\delta$ satisfies the triangle inequality, $\delta(A, C) \leq \delta(A, B)+\delta(B, C)$.

The function $\delta(A, B)$ can be rewritten as:

$$
\delta(A, B)=\sum_{x \in v(A) \cup v(B)}\left|m_{A}(x)-m_{B}(x)\right| .
$$

Since:

$$
\left|m_{A}(x)-m_{C}(x)\right| \leq\left|m_{A}(x)-m_{B}(x)\right|+\left|m_{B}(x)-m_{C}(x)\right|,
$$

the triangle inequality holds. Therefore $m t d$ is a metric on tree spaces.

\section{Naive Algorithm for computing mtd}

In what follows, we describe a naive algorithm for computing $\delta$ and $m t d$. Once $\delta$ is obtained, $m t d$ can be computed easily. In lines 3 to 7 of Algorithm 1, the tree $v$ is compared against all the trees of size $|v|$ of the multi-set $A$. This optimization is necessary to keep the complexity quadratic as we shall see later. The function $\delta$ (lines 12 to 14 ) calculates the intersection between the multi-sets $A$ and $B$ by obtaining the minimum of the multiplicity of the common elements. Finally in line 15 , the cardinality of the union of $\left|T_{1}\right|$ and $\left|T_{2}\right|$ is subtracted from the cardinality of the intersection of $T_{1}$ and $T_{2}$ and the result is returned to the caller.

We now proceed to analyze the complexity of algorithm $m t d$. The following lemma is useful: 


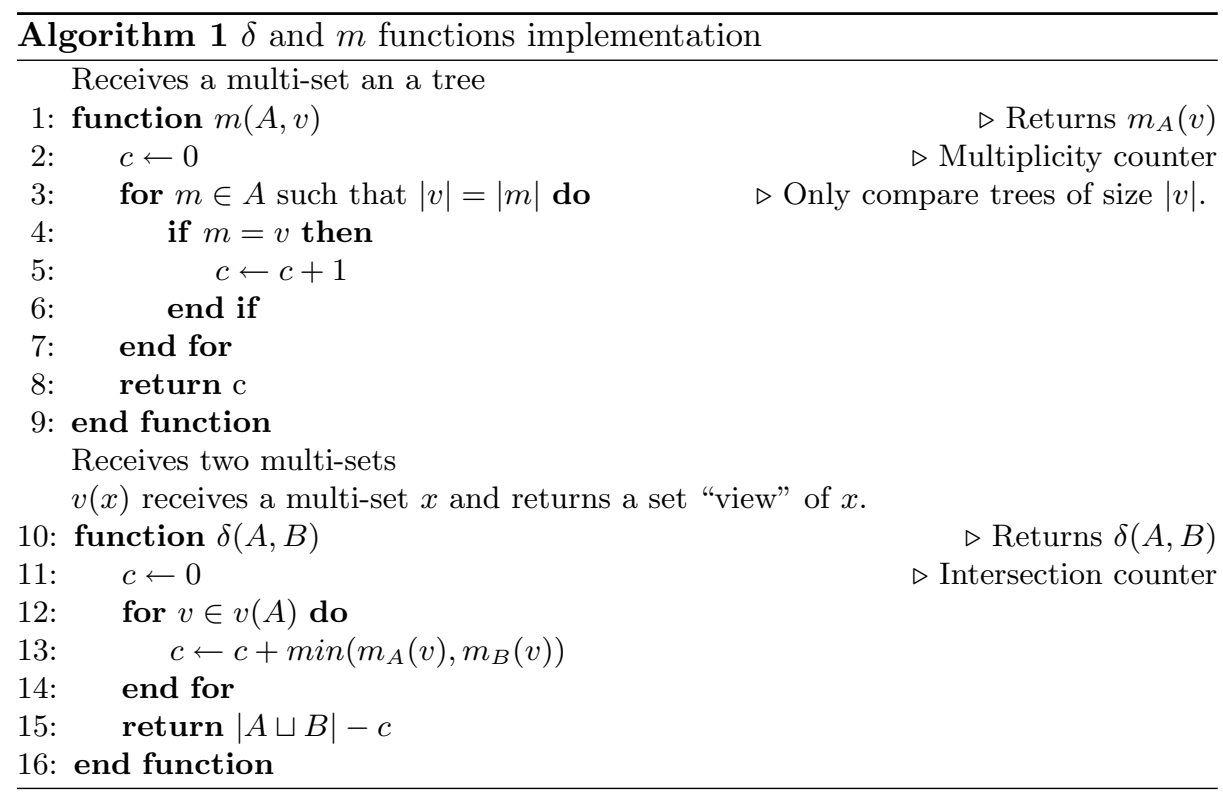

Lemma 1. Every tree of size $m$ has at most $\frac{m}{n}$ complete subtrees of size $n$.

Theorem 1. The distance function $m t d(F, G)$ can be computed in $O\left(\left|T_{1}\right| \times\left|T_{2}\right|\right)$ time and $O\left(\left|T_{1}\right|+\left|T_{2}\right|\right)$ space.

Proof. It is necessary to consider the cost involved in comparing the equality of two trees. For two trees $T_{1}$ and $T_{2}$, it is obvious that checking whether or not $T_{1}=T_{2}$, can be computed in $O\left(\left|T_{1}\right|\right)$ time. The computation of $s$ and $n$ can be achieved in linear time. Additionally, the space required by the multisets returned by $s$ and $n$ is linear because it is sufficient to store pointers to the original trees. Regarding $m$, we can see in line 3 of Algorithm 1 that for a complete subtree $v \in A$, the function will only compare complete subtrees of size $|v|$ in $B$.

Since $d_{n}$ matches only nodes (trees of size 1 ), it can be computed in linear time, therefore we will focus only on $d_{s}$. Each equality comparison for any complete subtree $v \in F$ will take at most $|v|$ steps, and will be executed at most $\frac{\left|T_{2}\right|}{|v|}$ times. This is because we will only match complete subtrees in $F$ of size $|v|$ (Algorithm 1, line 3) and because Lemma 1 guarantees that $\left|T_{2}\right|$ has only $\frac{\left|T_{2}\right|}{|v|}$ complete subtrees of size $|v|$. Therefore, the multiplicity $m_{s\left(T_{2}\right)}(v)$ can be computed in $O\left(|v| \times \frac{\left|T_{2}\right|}{|v|}\right)=O\left(\left|T_{2}\right|\right)$ time. Since the multiplicity function $m$ will be called by $\delta$ at most $\left|T_{1}\right|$ times, the function $\operatorname{mtd}\left(T_{1}, T_{2}\right)$ can be computed in $O\left(\left|T_{1}\right| \times\left|T_{2}\right|\right)$ time. Since it is possible to create a multi-set of pointers to the original nodes of the tree, the space complexity for $m t d\left(T_{1}, T_{2}\right)$ is $O\left(\left|T_{1}\right|+\left|T_{2}\right|\right)$. 
Our current implementation uses hash tables to improve performance. Hash codes have greater pruning power than the pruning by tree size described above, however this is not enough to lower the complexity of the algorithm. In the experiments of section 5 , we will see that in practice, our hash-based $m t d$ implementation matches the performance of a linear q-gram based distance function.

\section{Case Study}

In this section, we compare our function against BDist [16] and ted [15]. Currently, we are not able to run ted on our approximate program matching framework $^{1}$ because of its enormous computational cost. Therefore, our experiments were executed on real tree data extracted from program fragments[12] but focus on the distance functions only. The procedure to extract these fragments is detailed in [12]. Our data-set ${ }^{2}$ includes 244668 trees. The average depth is 4.75 , the average number of nodes is 11.11, and the number of nodes range between 1 to 20 nodes. In the experiment, we randomly selected 1000 trees (queries) from the data-set and compared them against the rest of the data-set. About 244 million tree comparisons were performed.

We implemented the ted [5] that can be computed in $O\left(n^{3}\right)$ time, $B$ Dist [16] that can be computed in $O(n)$ time, and $m t d$ that has a time complexity of $O\left(n^{2}\right)$. The distance functions were implemented in Java. The source code of the functions is available under the GPL license ${ }^{3}$. For the performance benchmarks, we loaded all the trees into memory and the time is counted after all the trees have been loaded. The experiments were executed on a Intel(R) Xeon(R) CPU $2.66 \mathrm{GHz}$ with 4 processors. Four threads were created to reduce execution time. Each thread performs the same job.

Regarding the $q$-gram function, we used Yang's BDist function[16]. In this case, BDist will create $|T|$ grams from a tree $T$ by creating for each node $v \in T$, a triple of the left child of $v, v$, and the right sibling of $v$. The original motivation of the paper was to create a similarity search engine by creating an inverted file of each q-gram. In this paper we simply calculated BDist in a sequential manner to show the real cost of a linear distance function. By using the similarity index presented by Yang, better performance results can be achieved for BDist. Finally, it can be proved that $B \operatorname{Dist}\left(T_{1}, T_{2}\right) \leq 5 \times \operatorname{ted}\left(T_{1}, T_{2}\right)[16]$.

\section{Distance Evaluation}

In Figure 3, we show the distribution of data according to distances between data and queries. In the $y$ axis, the percentage of calculations that returned the distance shown in the $x$ axis is displayed. We can see that all the functions maintain a similar, normal distribution.

\footnotetext{
${ }^{1}$ http://www. furiachan.org

${ }^{2}$ http://furia-chan.googlecode.com/files/trees1-20.tar.bz2

${ }^{3}$ http://furia-chan.googlecode.com/files/mtd-1.tar.bz2
} 
Distance Distribution

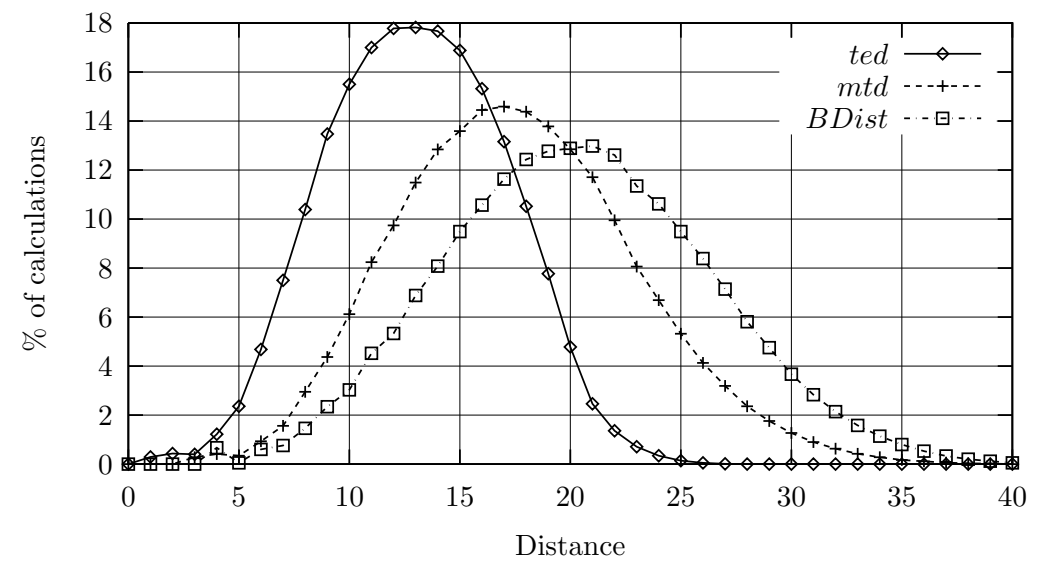

Fig. 3. Distribution of data according to distances between data and queries.

In Figures 4(a) and 4(b), we compare respectively, how $m t d$ and BDist vary from the result returned by ted. For $m t d$, the mean and standard deviation are closer than BDist. Additionally, maximum values for $m t d$ tend to be smaller than for BDist. On the other hand, Bdist's minimum values seem to be closer to ted. When BDist is used in its similarity search framework (and when a range $r$ is provided), additional pruning can be performed by the pre-order and post-order counts of the node. In this case, BDist cannot satisfy the triangle inequality and that is why we did not include this pruning. BDist is returning bigger results than $m t d$ because subtree movements return lower values than for BDist as siblings are not taken into account. For example in Figure 2, the distance returned by $B D i s t$ is 8 for $T_{1}$ and $T_{2}$. Since the shape of the distribution and the overall distance results are closer to ted, we can conclude that $m t d$ performs better than BDist.

In Figures 5(a) and 5(b), we compare how $d_{s}$ and $d_{n}$ vary from the result returned by $t e d$. We can see how $d_{s}$ has bigger maximum values than $m t d$ (Figure $4(\mathrm{a}))$. On the other hand, $d_{n}$ has smaller minimum values than $m t d$ or $d_{s}$. Finally, we can see that $m t d$ 's minimum and maximum range is improved by the combination of $d_{s}$ and $d_{n}$. The mean and standard deviation for $m t d$ is centered between $d_{s}$ and $d_{n}$. If we base our comparison on ted, the use of $d_{n}$ is justified. It is interesting to see that the closest function to ted when considering only the standard deviation is $d_{n}$.

\section{Benchmarks}

For the same experiment described at the beginning of the section, we recorded the execution time for ted, BDist and $m t d$. The function ted took about 85 hours to complete, BDist took 8 minutes, and $m t d$ took 7.4 minutes. The small difference between BDist and $m t d$ might be related to the hash function being 


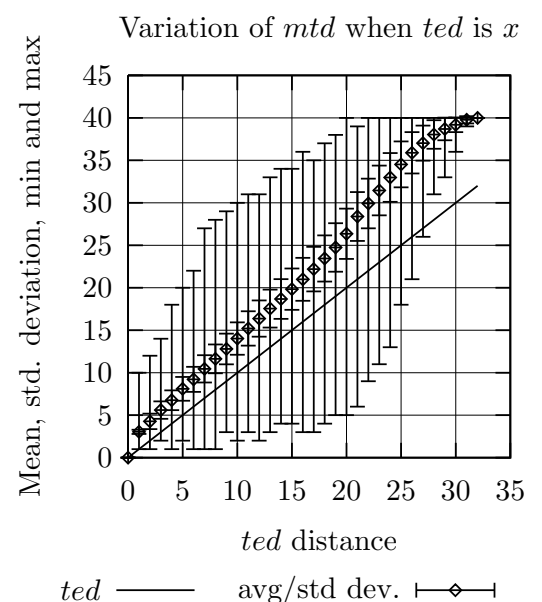

(a) $m t d$

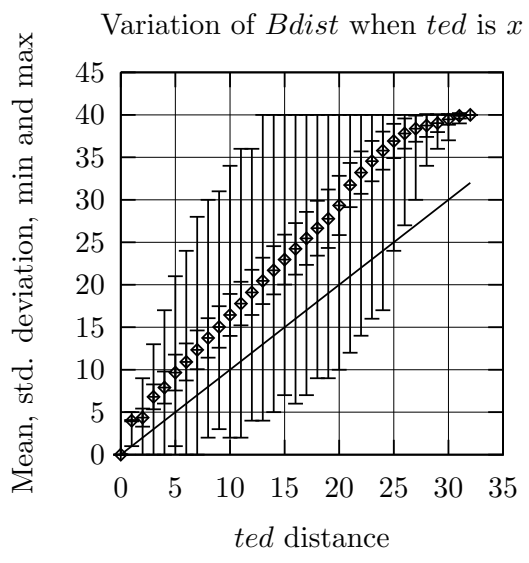

ted $\longrightarrow \quad$ avg/std dev. $\longmapsto$

(b) BDist

Fig. 4. Variation of $m t d$ and BDist with respect to the result $x$ of ted

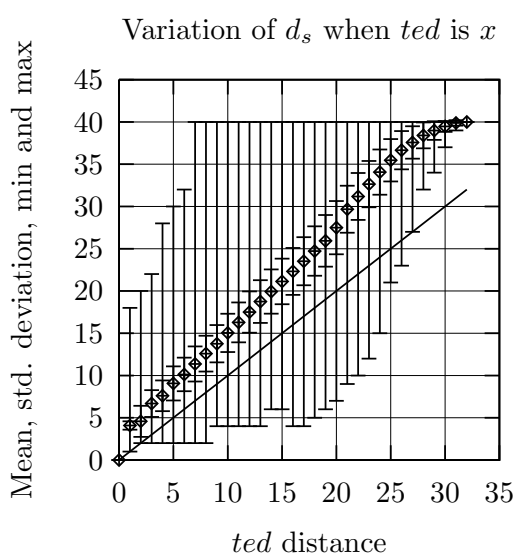

ted $\longrightarrow \quad$ avg/std dev. $\longmapsto$

(a) $d_{s}$

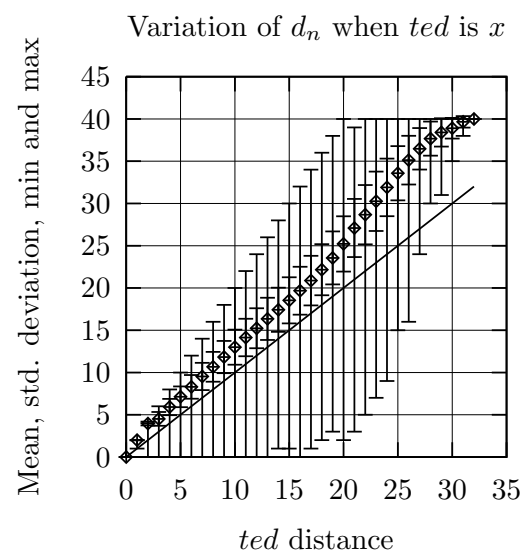

ted $\longrightarrow \quad$ avg/std dev. $\longmapsto$

(b) $d_{n}$

Fig. 5. Variation of $d_{s}$ and $d_{n}$ with respect to the result $x$ of ted 
called more times in BDist than in $m t d$. On the other hand, $m t d$ was implemented recursively and there is much room for improvement. One of the reasons ted is performing so poorly is that for each distance computation, many objects are being created (dynamic programming table objects and forests created and destroyed during the search). On the contrary, mtd and BDist only have to access some static structures that are computed once during the lifetime of a tree. From the benchmarking results, can see how the actual performance of our function seems to be on par with a linear q-gram distance function.

\section{Conclusions and Future Work}

We have introduced a tree distance function that is based on multi-sets. When compared to ted, our function is more sensitive to changes. This is in general an undesirable property, however the performance gains are considerable enough to make it a worthwhile candidate for matching trees. Our function is fast because it can perform matchings without resorting to expensive dynamic programming table memory allocations. Our hash table based implementation achieved similar performance than BDist[16], a q-gram based function that runs in time $O(n)$. Our distance function also has the added property of being a metric.

Regarding future works, because $m t d$ is faster than ted, it can be employed by techniques like SMAP [14] to find a subset of the data suitable for pivoting. From that subset, a set of pivots based on ted could be computed. Finally, we would like to study the effect of adding $\mathrm{N}$-grams greater than 1 to function $n$.

\section{References}

[1] N. Augsten, M. Bhlen, and J. Gamper. Approximate matching of hierarchical data using pq-grams. In $V L D B$ '05, pages 301-312, 2005.

[2] P. Bille. A survey on tree edit distance and related problems. Theoretical Computer Science, 337(1-3):217-239, 2005.

[3] S. S. Chawathe and H. Garcia-Molina. Meaningful change detection in structured data. SIGMOD Rec., 26(2):26-37, 1997.

[4] S. S. Chawathe, A. Rajaraman, H. Garcia-Molina, and J. Widom. Change detection in hierarchically structured information. SIGMOD Rec., 25(2):493-504, 1996.

[5] E. Demaine, S. Mosez, B. Rossman, and O. Weimann. An optimal decomposition algorithm for tree edit distance. In ICALP 'O7, $L N C S$, volume 4596, pages 146157. Springer-Verlag, 2007.

[6] M. Garofalakis and A. Kumar. Xml stream processing using tree-edit distance embeddings. ACM Trans. Database Syst., 30(1):279-332, 2005.

[7] T. Jiang, L. Wang, and K. Zhang. Alignment of trees - an alternative to tree edit. Theoretical Computer Science, 143(1):157-148, 1995.

[8] K. Kailing, H.-P. Kriegel, S. Schoenauer, and T. Seidl. Efficient similarity search for hierarchical data in large databases. In $E D B T$ ' $04 L N C S$, volume 2992, pages 676-693, 2004. 
[9] P. Klein, S. Tirthapura, D. Sharvit, and B. Kimia. A tree-edit-distance algorithm for comparing simple, closed shapes. In SODA '00, pages 696-704, Philadelphia, USA, 2000. Society for Industrial and Applied Mathematics.

[10] P. N. Klein. Computing the edit-distance between unrooted ordered trees. In ESA '98, LNCS, volume 1461, pages 91-102. Springer-Verlag, 1998.

[11] A. J. Müller-Molina and T. Shinohara. On approximate matching of programs for protecting libre software. In $C A S C O N$ '06, pages 275-289. ACM Press, 2006.

[12] A. J. Müller-Molina and T. Shinohara. Fast approximate matching of programs for protecting libre/open source software by using spatial indexes. In SCAM 'O', pages 111-122. IEEE Computer Society, 2007.

[13] N. Ohkura, K. Hirata, T. Kuboyama, and M. Harao. The q-gram distance for ordered unlabeled trees. In Discovery Science LNAI, volume 3735, pages 189-202, 2004.

[14] T. Shinohara and H. Ishizaka. On dimension reduction mappings for approximate retrieval of multi-dimensional data. In Progress in Discovery Science, LNCS, volume 2281, pages 224-231. Springer-Verlag, 2002.

[15] K.-C. Tai. The tree-to-tree correction problem. JACM, 26(3):422-433, 1979.

[16] R. Yang, P. Kalnis, and A. K. H. Tung. Similarity evaluation on tree-structured data. In SIGMOD '05, pages 754-765, 2005.

[17] K. Zhang. Algorithms for the constrained editing distance between ordered labeled trees and related problems. Pattern Recognition, 28(3):463-474, 1995.

[18] K. Zhang. Computing similarity between rna secondary structures. INTSYS '98, pages 126-132, 1998.

[19] K. Zhang, R. Statman, and D. Shasha. On the editing distance between unordered labeled trees. Information Processing Letters, 42(3):133-139, 1992. 